\title{
Early versus late sacral neuromodulation and systematic reviews
}

\author{
Sender Herschorn, MD, FRCSC
}

See related article on page 106

$\mathrm{n}$ this retrospective study from a single centre, ${ }^{1}$ the authors have demonstrated that earlier implantation of a neurostimulator for various types of "voiding dysfunctions" yields a better result than that seen with delayed implantation. ${ }^{1}$ Interestingly, this was consistent in both types of dysfunctions, urgency-frequency and urinary retention.

In another publication, Scheepens and colleagues, ${ }^{2}$ from the Netherlands, reviewed 211 of their patients who underwent percutaneous nerve evaluation to determine the clinical parameters that predict a positive response. They found that patients with urinary tract dysfunctions for a relatively long time have a significantly lower chance of a positive test compared with patients with dysfunction for a relatively short time. The cut-off point was less than 7 months versus 7 months or longer. The authors postulated that with sacral neuromodulation (SNM), the sensory and motor neural pathways of the lower urinary tract are probably being remodelled. They surmised that, if a disorder is present for a longer time, it may be more difficult to restore the normal balance between different reflexes. This is similar to the conclusions in the current study.

Neural plasticity is the capacity for continuous alteration of the neural pathways and synapses of the living brain and nervous system in response to experience or injury. ${ }^{3}$ In nonneurogenic voiding dysfunctions, reflexes become operative that interfere with normal function. For example, with urgency or detrusor overactivity, abnormal sensory afferent activity may lead to an abnormal micturition reflex, possibly mediated by a loss of higher centre voluntary control. With nonneurogenic nonobstructive retention, the guarding reflexes that usually prevent incontinence and that are inhibited by the brain during voiding may be abnormally active. SNM may activate or "reset" somatic afferent inputs that will modulate the micturition reflex via several mechanisms and restore normal function. Pudendal afferent input from SNM may turn on voiding reflexes by suppressing the guarding reflex pathways and can turn off supraspinally mediated hyperactive voiding by blocking ascending sensory pathway inputs. ${ }^{4}$

The question is, does SNM work, and if so, for how long? The Ontario Ministry of Health and Long-Term Care conducted a technology assessment on SNM and reviewed international health technology assessments and English-language journal articles published from 2000 to November 2004, in addition to new unreported trials. ${ }^{5}$ They reported on 4 previous technology assessments: the National Institute for Clinical Excellence from Britain, the Australian Safety and Efficacy Register of New Interventional Procedures, the Medical Services Advisory Committee in Australia and the Blue Cross Blue Shield in the United States. These assessments consistently reported that SNM was an effec- tive technology for managing urge incontinence, urgency-frequency and urinary retention in patients who did not respond to drug or behaviour therapy. Complication rates ranged from $33 \%-50 \%$, but there were no incidences of permanent injury or death.

The Ontario assessment reviewed 2 randomized clinical trials (RCTs) in patients with refractory urge incontinence; both showed significant improvements in voiding function. Three studies (1 RCT, 1 retrospective review and 2 prospective case series) showed significant improvement for urgency-frequency with or without chronic pelvic pain. One RCT and 1 case series also showed significant improvement for refractory urinary retention. Of 6 studies identified describing long-term follow-up, the follow-up periods ranged from 1.5 years to over 5 years. The success rate of $\geq 50 \%$ improvement in voiding function was 58\%$77 \%$. Explantation rates were $12 \%-21 \%$. Overall, there was a 33\% surgical revision rate, and no serious injury or death was reported.

The Ontario assessment concluded that there is level 2 evidence to support the effectiveness and safety of SNM for patients with refractory urge incontinence, urgency-frequency or urinary retention. This is a very high level of evidence, compared with many other surgical procedures. The document also outlined the number of Ontario residents who might benefit from SNM. Between 3842 and 12565 individuals may qualify for SNM and 1263 to 6283 may have a successful stimulation test. Currently, there is only 1 site in Ontario where SNM is performed - the Toronto Western Hospital — and it is restricted to 12 patients per year. In the rest of Canada, there are 5 centres in 3 provinces.

This article adds information about the natural history of untreated voiding dysfunction. Although there is still much to be learned about the mechanism of action of this tech- 
nology, there is evidence of the benefit and a huge unmet need. Clearly, much more work must be done to expand this technology in Canada.

Professor and Chair, Division of Urology, University of Toronto, and Martin Barkin Chair in Urological Research and attending urologist, Sunnybrook Health Sciences Centre, Toronto, Ont.

Competing interests: None declared.

\section{References}

1. Hassouna MM, Elkelini MS. Early versus late treatment of voiding dysfunction with pelvic neuromodulation. CUAJ 2007;1 (2):106-10.
2. Scheepens WA, Jongen MM, Nieman FH, et al. Predictive factors for sacral neuromodulation in chronic lower urinary tract dysfunction. Urology 2002;60:598-602.

3. Plasticity. Merriam-Webster Online. Available: www.m-w.com/dictionary/plasticity. Accessed May 10, 2007.

4. Leng WW, Chancellor MB. How sacral nerve stimulation neuromodulation works. Urol Clin North Am 2005;32:11-8.

5. Medical Advisory Secretariat. Sacral nerve stimulation for urinary urge incontinence, urgency-frequency, urinary retention, and fecal incontinence. Toronto: Ontario Ministry of Health and Long Term Care; 2005.

Correspondence: Dr. Sender Herschorn, Division of Urology, University of Toronto, Sunnybrook Health Sciences Centre, Rm. A309, 2075 Bayview Ave., Toronto ON M4N 3M5; S.Herschorn@utoronto.ca

\section{$\sqrt{6}$ \\ Symposium \\ June 27, 2007, 7:00 am - 7:50 am \\ During Canadian Urological Association \\ Annual Meeting \\ Quebec City \\ “Optimizing Prostate Health: Practical take- aways from PCPT and MTOPS in the prevention, screening and treatment of LUTS-BPH and early prostate cancer".}

\section{Chair:}

Dr. Laurence Klotz

Sunnybrook \& Women's College HSC

Faculty:

Dr. Armen G. Aprikian

MUHC - Montreal General Hospital

Dr. Pierre I. Karakiewicz

CHUM - Hôpital St-Luc

Dr. Laurence Klotz

Sunnybrook \& Women's College HSC 